\title{
HIGH-PRECISION ASTROMETRY AND THE MODELLING OF STELLAR SPECTRA
}

\author{
BENGT GUSTAFSSON \\ Uppsala Astronomical Observatory \\ Box 515 \\ S-751 20 Uppsala \\ Sweden
}

\begin{abstract}
The impact of high-precision astrometry on modelling of stellar spectra and determination of fundamental stellar parameters for single stars is discussed. It is found to be considerable, but improvements in the understanding of stellar atmospheres, and in particular of the physical background of luminosity (i.e., surface-gravity) criteria will be needed in order to match the possibilities of stellar parallaxes with accuracies on the order of a few percents or better.
\end{abstract}

\section{Introduction}

It is an interesting fact that the organisers of this symposium have planned a discussion of the possibilites of modelling stellar atmospheres and spectra. This demonstrates the connections between stellar positional astronomy and astrophysics, revitalized by the remarkable developments discussed, in particular by the possibility of getting accurate parallaxes for a very great number of stars. I shall restrict this presentation to single stars, since Johannes Andersen will later discuss the situation for binary stars.

\section{Direct determination of stellar radius and mass}

A first task is to use the parallaxes, $\pi$, together with photometry to derive stellar radii, $R$, according to

$\log R=-\log \pi-2 \log T_{\text {eff }}-0.2 V+0.2 A_{v}-0.2$ B.C. + const.

Obviously, if we wish to match an accuracy of, e.g., $5 \%$ in the parallaxes, we need to establish the effective temperatures $\mathrm{T}_{\text {eff }}$ to about $2 \%$. This can be achieved, both for lateand early-type stars today, using model-atmosphere techniques. For the late-type stars, one may use colours, properly calibrated (see, e.g. Edvardsson et al. 1993, Buser and Kurucz 1992, Bell et al. 1994), or the "infrared flux method" (see, e.g. Blackwell et al. 1991, Bell 
and Gustafsson, 1989) which is less model dependent, or profiles of hydrogen lines (Cayrel et al. 1985, cf. also Fuhrmann et al. 1993). For the hot stars fits to model atmosphere spectra of suitable spectral line profiles seem to give the most satisfactory results (cf. Kudritzki and Hummer, 1990). (An interesting question is whether the effective-temperature determination could be made with an accuracy of about $1 \%$ - a figure claimed as an internal error in several contemporary studies. This seems indeed possible, but further work is needed before such an accuracy can be regarded as well established in any temperature interval.) In addition, interstellar extinction $\left(A_{v}\right)$ and bolometric correction (B.C.) need to be known to an accuracy of about $0 \mathrm{~m}^{\mathrm{m}} 1$. The latter quantity is also derived from model atmospheres, however, for all stars but the hottest and the coolest ones there should not be any severe problem to estimate it with this accuracy.

Knowing the radius, one could estimate the stellar mass $M$ from the spectroscopically determined surface gravity, $g=G M / R^{2}$ ( $G$ being Newton's contant of gravity). I.e.,

$\log \mathrm{M}=\log \mathrm{g}-2 \log \pi-4 \log \mathrm{T}_{\text {eff }}-0.4 \mathrm{~V}+0.4 \mathrm{~A}_{\mathrm{v}}-0.4$ B.C. + const.

The dominating problem here is to estimate the surface gravity. For this one uses pressuresensitive spectral features, such as the strength of spectral lines from ions relative to neutral atoms, molecular lines, wings of pressure-broadened strong lines, or pressure-sensitive features in the continuous flux distribution, such as the Balmer discontinuity in F star spectra. These different methods have been studied and compared in recent work, e.g. by Bonnell and Bell (1994). We note in passing that, in fact, stellar spectra give knowledge about the gravity scaled by a function of the helium abundance Y (cf. Strömgren et al. 1982). This dependence is, however, so weak for a realistic variation of $Y$ that one may hardly hope for interesting bounds on Y resulting from this effect - see, however, the comments on the "Hyades anomaly" below.

A general conclusion from these studies is that it is very difficult to reach an accuracy better than about 0.2 in log $g$ determinations from spectral lines for late-type stars, while for early-type stars an accuracy of about 0.1 in $\log \mathrm{g}$ can be reached. The problems are due to different reasons, one being the non-LTE effects affecting ionization and possibly molecular equilibria and often more difficult to model in late-type stars, another being the lack of adequate atomic and molecular data (such as damping constants), a third being the strong temperature sensitivity of molecular equilibria, which leads to severe effects from uncertainties in effective temperatures and temperature structures, a fourth being crowded spectra which makes it difficult to trace extended line wings.

An interesting group of stars for which more accurate log $\mathrm{g}$ determinations are possible are the main-sequence stars of spectral types F - early G. Edvardsson et al. (1993) have shown that the Balmer discontinuity index $\delta c_{1}$ of the Strömgren four-colour photometry may be used with a semiempirical calibration for deriving $\log g$ for these stars with errors in the interval 0.05 - 0.10 dex. A prevailing problem here is the "Hyades anomaly" (Strömgren et al. 1982, Nissen 1988) which indicates that some as yet unknown effects on the $\delta c_{1}$ index set a limit on the accuracy obtainable. It seems that the good fit between stellar models and the observed colour-magnitude diagram and $\mathrm{Li}$ abundances for the Hyades rule out a severe He abundance anomaly as a reason for this phenomenon (cf. Swenson et al. 1994). 
We conclude that one cannot expect to derive masses for individual stars from Eq (2) with an accuracy better than about 0.2 dex for general late-type stars in the field; for early-type stars the similar error is about 0.1 dex, provided that accurate parallaxes may be obtained. For solar-type dwarfs, one may get even somewhat more accurate masses than that.

\section{Parallaxes and checks on spectroscopic estimates}

The difficulties mentioned in spectroscopic gravity determination for late-type stars make, however, other applications of accurate parallaxes interesting. For certain types of stars, e.g., the very cool giants and dwarfs, the art of determining spectroscopic gravities is very difficult or undeveloped. This causes trouble in spectroscopic analyses. E.g., in our study of carbon stars, based on highly resolved infrared spectra (Lambert et al. 1986), we found that errors in $\log \mathrm{g}$, estimated to $0.5 \mathrm{dex}$, were of major significance for the uncertainties in the resulting abundances. In this case the gravities were not estimated from the spectra but from mass estimates, based on different astrophysical arguments, and from estimated absolute magnitudes. With direct and relatively accurate parallaxes for these stars (we note that the proposed Roemer mission would produce parallaxes better than $1 \%$ for hundreds of carbon stars), one could reduce the uncertainty in the spectroscopic analyses to that reflecting the uncertainty in the masses.

In this connection one should note that spectra of late-type $\mathrm{M}$ and $\mathrm{N}$ giants and supergiants in fact contain not only information on mass and radius in the combination $g=G M / R^{2}$ but also, which is a consequence of the spherical atmospheric structure, on $M$ and $R$ individually, as was first noted by Schmid-Burgk et al. (1981). Attempts to determine both these parameters from spectroscopy have, however, not given any reliable results yet, due to the complexity of the situation, including the fact that the extension of these tenuous atmospheres is not only the result of radition transfer and hydrostatic equilibrium - velocity fields are probably also of decisive importance for the structure. Here, accurate parallaxes will overdetermine the parameter problem; in principle radii will be known and that should help in the mass determination and give a useful consistency check. Also, for stars with measured angular diameters for different wavelengths, the accurate parallaxes give checks on the limb-darkening predictions from the model atmospheres and the absolute extension of the atmospheres.

For hot stars with radiatively driven and reasonably stationary winds Kudritzski and collaborators (cf. Kudritzski and Hummer 1990) have shown that the observed terminal wind velocities can be used for estimating the escape velocity $\left(\alpha(M / R)^{1 / 2}\right)$ and thus, with the spectroscopically determined surface gravity $\left(\alpha M / R^{2}\right)$ both $M$ and $R$ may be determined spectroscopically. This can then be used for deriving spectroscopic parallaxes, offering a method to determine distances to nearby galaxies. Again, comparisons with accurate trigonometric parallaxes for hot stars, in our Galaxy, may give further checks on the method and thus on the basic assumptions behind it. 


\section{When evolutionary tracks are also used}

What is said above is, as regards astrophysics, in principle only related to our understanding (or lack of understanding) of stellar atmospheres. However, we also have considerable knowledge about stellar structure and evolution. Naturally, a good deal more may be learnt about the stellar parameters from parallaxes and spectra if this knowledge is also added. I will not go deeply into that, since it will be covered in several other contributions at this symposium - just add a few remarks on the significance and shortcoming in the understanding of stellar atmospheres in this respect.

In principle, the radius and luminosity of a star are functions of its mass, age, chemical composition $(\mathrm{Y}, \mathrm{Z}, \ldots)$ and possibly angular momentum. Measuring the stellar luminosity (via the parallax), radius (via $\mathrm{T}_{\text {eff }}$ and Eq. (1)), and estimating $\log \mathrm{g}$ and $\mathrm{Z}$ spectroscopically, one may ask with which accuracy age and, say, helium abundance may now be derived. Clearly, the answer is very different for different regions in the HR diagram. For dwarfs of low mass, presumably close to the ZAMS, the helium abundance (Y) may be directly determined from luminosity and effective temperature, provided that the heavy-element abundance is satisfactorily known. In fact, $\mathrm{Z}$ should be determined from stellar spectra with an accuracy of about 0.1 dex in order to enable a determination of $Y$ with an accuracy better than 0.03 for disk stars (cf. Perrin et al. 1977). Similarily, Teff must be known to $1 \%$ in order to allow an accuracy of 0.03 in the $Y$ determination. We see that the problem of estimating $\mathrm{Y}$ to an interesting accuracy puts rather heavy demands on the quantitative interpretation of stellar spectra, at least if not a strictly differential approach can be used for minimizing the errors.

For solar-type stars in the main-sequence band but at some distance from the ZAMS (in order to avoid the "degeneracy" caused by the early evolution almost along the ZAMS in the $\log$ L-T $\mathrm{T}_{\text {eff }}$ diagram) one may derive evolutionary ages with an accuracy of about $20 \%$, using uvby photometry with a semiempirical calibration (Edvardsson et al. 1993), provided that a helium abundance is adopted. Good parallaxes give an interesting check on these ages. In order to trace effects corresponding to the Hyades anomaly, however, they have to be accurate to at least $3 \%$.

One may ask whether it would be possible to separate the effects of age from those of the He abundance for individual stars and thus determine both quantities from $\mathrm{L}, \mathrm{T}_{\text {eff }}$ and $\log$ g. Using the evolutionary tracks of Hejlesen (1980) I have found that the very high accuracy in $\log g$ of about 0.03 would be needed to bring down the error in $\mathrm{Y}$ to 0.03 . Obviously, for single stars we are very far from reaching the accuracy in various parameters that one may obtain for visual binaries.

An interesting group of stars for which stellar ages and masses may be determined relatively accurately from good parallaxes, photometric data and evolutionary tracks are the subgiants with relatively low masses at temperatures so high $\left(\mathrm{T}_{\text {eff }}>5500 \mathrm{~K}\right)$ that they are still not on the giant branch where the evolutionary tracks merge. Thus, with parallaxes (giving luminosities by means of photometric data) accurate to $5 \%$ one may derive ages with an accuracy better than $20 \%$ for these stars, provided that the metal abundances are determined to 0.1 dex. This also assumes that the helium abundance $\mathrm{Y}$ is known within 
0.05 and that the convection parameter $\left(1 / \mathrm{H}_{\mathrm{p}}\right)$ is known or constant with an accuracy of about 0.5 . This was estimated from the standard model sequences; if, instead convective overshoot is allowed (using the tracks of Dowler and VandenBerg 1994, kindly supplied by the latter) similar conclusions may be drawn as long as the degree of overshoot behaves regularily (i.e. is constant or smoothly varying with the stellar fundamental parameters: mass, age and chemical composition). These ages and masses are not very dependent on the effective-temperature estimate, since the evolutionary tracks are almost horizontal in this part of the HR diagram. Thus, the distribution of stellar ages, masses and metallicities could be determined from Hipparcos parallaxes for these stars, without relying on stellar spectroscopy or narrow-band photometry except when estimating the metallicities.

Also for red giants the Hipparcos data, and parallaxes with higher accuracy from possible future missions for stars that are more rare, will contribute important but rather indirect statistical evidence on the stellar physical properties. Using Eq. (1) and an adopted mass distribution, e.g. derived from an observationally derived Initial Mass Function and an assumed star-formation rate, one may empirically calibrate measures of surface gravities. These may then be compared with theoretical calibrations, based on model atmospheres and synthetic spectra, and conclusions may be drawn concerning the adequacy of the modelling or concerning stellar mass loss. The study of binary stars or cluster giants offer similar, less statistical, tests. In passing, we note that current mass loss estimates for cool giants, based on observations of $\mathrm{CO}$ millimetre line or IR dust emission, are strongly dependent on the distance estimate (cf. Olofsson et al. 1993); in this case accurate parallaxes will considerably improve the situation.

\section{Conclusions}

We have found that accurate parallaxes for nearby single stars may significantly improve our determinations of stellar radii, contribute to better determinations of stellar masses and surface gravities, will improve stellar age estimates and even enable rough heliumabundance estimates for some types of stars. However, when the errors in parallaxes decrease below about $5 \%$, basic uncertainties in the interpretation of stellar fluxes and spectra will often limit the degree to which the astrometric accuracy may be fully used for deriving fundamental stellar parameters. Instead, such parallaxes may in some cases contribute to our basic knowledge about the physics of stellar atmospheres.

ACKNOWLEDGEMENTS. Bernard Pagel is thanked for valuable comments on an earlier version of this manuscript, Don VandenBerg for supplying new evolutionary tracks and Giusa Cayrel de Strobel for interesting discussions.

\section{REFERENCES}

Bell, R.A., Gustafsson, B.: 1989, MNRAS, 236, 653

Bell, R.A., Paltoglou, G., Tripicco, J.: 1994, MNRAS, 268, 771

Blackwell, D.E., Lynas-Gray, A.E., Petford, A.D.: 1991, A\&A, 245, 567

Bonnell, J., Bell, R.A.: 1993, MNRAS, 264, 334

Buser, R., Kurucz, R.L.: 1992, A\&A, 264, 557

Cayrel, R., Cayrel de Strobel, G., Campbell, B.: 1985, A\&A, 146, 249 
Dowler, P., VandenBerg, D.: 1994, in preparation

Edvardsson, B., Andersen, J., Gustafsson, B., Nissen, P.E., Lambert, D.L., Tomkin, J.: 1993, A\&A, 275, 101

Fuhrmann, K., Axer, M., Gehren, T.: 1993, A\&A, 271, 451

Hejlesen, P.M.: 1980, A\&AS, 39, 347

Kudritzki, R.P., Hummer, D.G: 1990, ARA\&A, 28, 303

Lambert, D.L., Gustafsson, B., Eriksson, K., Hinkle, K.H.: ApJS, 62, 373

Nissen, P.E.: 1988, A\&A, 199, 146

Olofsson, H., Eriksson, K., Gustafsson, B., Carlström, U.: 1993, ApJS, 87, 267

Perrin, M.-N., Hejlesen, P.M., Cayrel de Strobel, G., Cayrel, R.: 1977, A\&A, 54, 779

Schmid-Burgk, J., Scholz, M., Wehrse, R.: 1981, MNRAS, 194, 387

Strömgren, B., Olsen, E.H., Gustafsson, B.: 1982, PASP, 94, 5

Swenson, F.J., Faulkner, J., Rogers, F.J., Iglesias, C.A.: 1994, ApJ, 425, 286. 